\title{
Glottopol
}

Revue de sociolinguistique en ligne

$35 \mid 2021$

La langue à l'école, de l'institution à la classe : quelles conceptions, quelles normes, pour quels usages?

\section{Variation et approche polynomique : pour une conception plurielle de la langue à l'école calédonienne}

Claire Colombel-Teuira et Véronique Fillol

\section{CpenEdition}

\section{Journals}

Édition électronique

URL : https://journals.openedition.org/glottopol/286

DOI : $10.4000 /$ glottopol.286

ISSN : 1769-7425

Éditeur

Presses universitaires de Rouen et du Havre

Référence électronique

Claire Colombel-Teuira et Véronique Fillol, « Variation et approche polynomique : pour une conception plurielle de la langue à l'école calédonienne », Glottopol [En ligne], 35 | 2021, mis en ligne le 01 janvier 2021, consulté le 02 octobre 2021. URL : http://journals.openedition.org/glottopol/286 ; DOI : https:// doi.org/10.4000/glottopol.286 


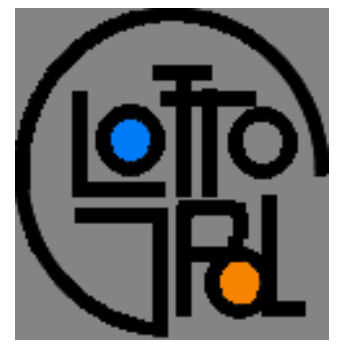

\section{GLOTTOPOL}

Revue de sociolinguistique en ligne $\mathrm{n}^{\circ} 35$ - janvier 2021

\section{La langue à l'école, de l'institution à la classe : quelles conceptions, quelles normes, pour quels usages?}

\section{SOMMAIRE}

Catherine Delarue-Breton et Élisabeth Bautier : Introduction

Catherine Delarue-Breton : Des conceptions scolaires de la langue en tension : l'exemple du programme de 2015 pour l'école primaire et le collège

Véronique Miguel-Addisu : "Sport de riche je l'ai changé en sport élitiste » : plurilinguisme et variation dans la langue de l'école du point de vue des élèves

Claire Colombel-Teuira, Véronique Fillol : Variation et approche polynomique : pour une conception plurielle de la langue à l'école calédonienne

Daphné Bloch : Pratiques langagières, situations pédagogiques et construction d'inégalités d'apprentissage à Madagascar

Samatar Abdallah Doualeh : Le français langue de l'école djiboutienne ou l'hégémonie de la norme exogène

Élise Vinel, Élisabeth Bautier : Des discours des élèves sur l'orthographe aux pratiques des enseignants, analyse d'entretiens métagraphiques

Thierry Pagnier, Belinda Lavieu-Gwozdz: Regards sur le discours scolaire: saisir des conceptions de la langue et de son enseignement

Jacques Crinon, Georges Ferone, Hélène Font : Les enseignants et l'orthographe, une enquête au cycle 3

Christel Troncy : Les enseignants, la norme scolaire et la pluralité langagière dans deux écoles immersives à programme français en Californie. Dynamiques des attitudes et des pratiques lors d'une recherche collaborative

Erwan Le Pipec: L'école, planche de salut du breton?

Argia Olçomendy : La langue basque dans les instructions officielles (1976-2019)

\section{Compte rendu de lecture}

Par Katrin Pfadenhauer: Ursula Reutner (Ed.), 2017, Manuel des francophonies, Berlin/Boston, de Gruyter, 745 p.

Par Robert Fournier: Shana Poplack, 2018, Borrowing: Loanwords in the speech community and in the grammar. New York: Oxford University Press ; xxi, $246 \mathrm{p}$.

Par Marie-Jeanne Verny: Catherine Adam, 2020, Bilinguisme scolaire. Familles, écoles, identités en Bretagne, éd. Peter Lang, coll. «Langue, multilinguisme et changement social », Berlin. 


\title{
VARIATION ET APPROCHE POLYNOMIQUE : POUR UNE CONCEPTION PLURIELLE DE LA LANGUE À L'ÉCOLE CALÉDONIENNE
}

\author{
Claire Colombel-Teuira* et Véronique Fillol** \\ *Institut Universitaire de Formation des Maitres de Nouvelle-Calédonie et \\ **Université de la Nouvelle-Calédonie, ERALO
}

Comment l'école calédonienne construite sur le modèle (français) d'une école républicaine monolingue $^{1}$, utilisée comme instrument privilégié et efficace d'unification et de domination linguistiques, peut-elle prendre en compte la diversité linguistique et culturelle de ses élèves pour la réussite de tous ${ }^{2}$ ? En d'autres termes, comment l'école calédonienne d'aujourd'hui peut-elle aider les élèves à se construire comme sujets plurilingues tout en s'appropriant la langue de scolarisation? Comment former les enseignants à une didactique du plurilinguisme ou a minima à une didactique du français contextualisé dans un contexte où le plurilinguisme n'est pas " politiquement assumé » comme c'est le cas en Corse (Cortier et Di Meglio, 2018) ou en Polynésie française ? (Vernaudon, 2020). Comment traiter les usages langagiers des élèves ? Comment accueillir en classe, voire traiter didactiquement la variation linguistique ${ }^{3}$ ?

En Nouvelle-Calédonie, ces questions se sont avérées centrales dans nos activités d'enseignantes-chercheures (formatrices) en sociolinguistique et didactique du français contextualisé. Elles nous ont conduites à développer des travaux sur une période de 20 ans tout en renouvelant nécessairement les concepts, méthodes, et postures de recherche (ColombelTeuira \& Fillol, 2020). Nous précisons par ailleurs que nous avons privilégié une approche systémique et compréhensive des questions langagières partant du principe que «pour produire du changement réel, et avancer vers une école plus inclusive, il vaut mieux soutenir l'existant plutôt que de prescrire l'idéal ${ }^{4}$.

\footnotetext{
${ }^{1}$ Dès le début l'administration coloniale du Territoire, les outils de domination de la langue française sur les langues kanak ont petit à petit été mis en œuvre ; depuis les décrets interdisant l'usage (oral à l'école et écrit en toute circonstance) des langues kanak jusqu'à l'objectif principal des écoles indigènes : "Les écoles indigènes sont destinées à propager l'usage du français parmi les indigènes et à les initier à la pratique d'un métier. Elles sont donc surtout des écoles de langage et d'apprentissage » (Décret du 26 septembre 1902, article ${ }^{\text {er }}$ ).

${ }^{2}$ Cette question a été régulièrement retenue comme thème des débats sur l'avenir de l'école calédonienne.

${ }^{3}$ Nous faisons l'hypothèse à l'instar de $\mathrm{S}$. Clerc-Conan et $\mathrm{C}$. Richerme-Manchet que « le rapport à la langue de l'école se modifie grâce au sens donné à la variation linguistique (laquelle n'est plus vue en termes de «niveaux de langue » $(2016: 55)$.

4 http://centre-alain-savary.ens-lyon.fr/CAS/education-prioritaire/ressources/theme-4-perspectives-relatives-aupilotage-et-a-levaluation/francoise-lantheaume-coordination-regulation-cooperation-quels-defis-pour-lesmetiers-en-education-prioritaire
} 
Il s'agit pour nous d'envisager les questions centrales d'articulation entre les langues (et les différentes variétés de ces langues) mais aussi des fonctions des langues dans le cadre scolaire.

La question des attitudes inclusives (ou exclusives) des pratiques langagières (et de la variation sociolangagière) est une question vive (en France ${ }^{5}$ et plus largement dans la francophonie) au cœur de tensions et d'injonctions paradoxales (et mouvantes selon les programmes scolaires).

Ainsi, le point commun aux recherches-actions (Colombel-Teuira, Fillol \& Bousquet, 2020) ou aux projets pédagogiques que nous accompagnons sont de participer à déconstruire le paradigme monolingue (et mononormatif) et de partir des besoins exprimés par les enseignants, élèves ou étudiants.

Pour suivre le fil esquissé par le titre de ce numéro thématique «la langue de l'école, de l'institution à la classe », la présente contribution s'articulera en trois parties: nous présenterons succinctement le contexte sociolinguistique dans lequel les contacts ou rapports aux langues sont ambivalents. Les parties suivantes seront consacrées à des outils (socio)didactiques visant à accompagner les enseignants à observer autrement les usages langagiers des élèves et à une étude de cas d'une recherche-collaborative qualifiée d'approche polynomique.

\section{Des recherches situées : contexte institutionnel et sociolinguistique}

\section{Un pays multilingue à langue dominante unique : le français seule langue de scolarisation}

Les différentes descriptions de la diversité linguistique néo-calédonienne sont relatives aux discours ou instances qui les produisent. Ainsi les linguistes et l'Académie des langues kanak présentent la diversité endémique en dénombrant $28^{6}$ langues kanak - réparties en huit aires coutumières et qualifiées de langues de France. Les sociolinguistes donnent de la diversité linguistique calédonienne, une représentation plus large qui inclue les variétés de français et des langues kanak, les langues issues de migrations proches (langues océaniennes telles que le wallisien et le futunien, les langues de Polynésie française, les langues indonésiennes...) et de migrations plus lointaines, ou encore les langues importées par les acteurs de l'industrie minière (Morel-Lab, 2014).

Pour le contexte calédonien, si on peut - et c'est même une nécessité, voire une obligation politique et éthique - interroger les finalités de l'enseignement des langues kanak, il nous semble pertinent de ré-interroger aussi les finalités de l'enseignement du français en situation multilingue (Fillol, 2009, 2016a). Élisabeth Bautier (2004) préconisait, à propos de la situation française, de revenir sur ce qui est souvent flou quand on parle de «maitrise de la langue » parce que l'École, elle-même, est sans doute aujourd'hui le lieu d'une confusion concernant les objectifs de l'enseignement du français. Précisément, cette question des objectifs de

\footnotetext{
${ }^{5}$ La littérature est abondante sur cette question dans le champ de la sociologie du langage, de la sociolinguistique, de la didactique et des sciences de l'éducation. Pour prendre un exemple, Claudine Dannequin avait réalisé il y a plus de trente ans des enregistrements et analyses de moments de « classe » de langage en cours préparatoire, pour étudier les attitudes linguistiques dans trois classes parisiennes. Elle en conclut que l'usage du langage à l'école s'avère très différent de la production ordinaire. Le maitre ne dit pas ce qu'il attend des élèves, et à la limite, peu importe ce que disent les élèves du moment qu'ils le disent «bien »-d'où des malentendus sur les attentes, les objectifs, les tâches à réaliser du point de vue de l'élève. Les situations de langage permettent mal de repérer les difficultés linguistiques des élèves, les productions devant se couler dans le moule de phrases courtes et «simples » (sujet-verbe-objet). Nous avons lors de visites de classe observé les mêmes attitudes et gestes professionnels : à titre d'exemple un échange de 15 minutes entre un enseignant et un élève, l'enseignant attendant (exigeant) que l'élève produise LA réponse « attendue ».

${ }^{6}$ L'Atlas interactif des langues en danger de l'Unesco compte 16 langues kanak menacées, dont 5 sérieusement en danger ou en situation critique (Moseley, 2010).
} 
l'enseignement du français, souvent flous ou fluctuants, qui peuvent diverger selon les acteurs (politiques, éducatifs...) se pose autrement et avec force, dans les départements d'outre-mer.

Dans cette situation que nous avons qualifiée de «pluridiglossique », le français est l'unique langue officielle de la République et en dépit de l'Accord de Nouméa qui fait des langues kanak "des langues d'enseignement et de culture », la langue française «reste la langue de scolarisation » (RAZAFI \& Favard, 2018 : 313). Si l'on peut rencontrer des personnes âgées de 60 ans (ou plus) vivant en NC ne parlant pas le français, celui-ci est présent dans le répertoire langagier de tous les élèves, cependant, "l'écart entre le français couramment pratiqué hors contexte d'apprentissage scolaire et celui mononormatif de l'école peut s'apparenter, aux yeux des élèves, à deux langues différentes. » (ibid.).

Par ailleurs l'introduction des langues $\operatorname{kanak}^{7}$ dans le système scolaire calédonien (programmes scolaires votés en septembre 2005) n'a pas modifié les fonctions assignées à la seule langue de scolarisation: "ses missions et ses fonctions sont multiples" nous dit le Rapport du Grand Débat :

- la langue française est d'abord la langue de scolarisation ; sa maitrise est indispensable à chaque étape du parcours scolaire ;

- la langue française, langue véhiculaire, permet la communication orale et écrite entre toutes les communautés qui constituent la Nouvelle-Calédonie ; elle constitue à ce titre un outil essentiel à la construction d'un destin commun : une langue partagée favorise le vivre ensemble. (Commission du Grand Débat, 2011)

La tradition plurilingue des sociétés océaniennes a donc été remplacée - en partie grâce à la massification de la scolarisation - par une idéologie monolingue. Dans cette perspective, l'école est le lieu de diffusion et d'apprentissage de la langue française, "langue partagée ", nous disent les recommandations, censée assurer et garantir la civilité de tous les citoyens et l'entente entre les peuples :

Précisément, si le français et ses variantes cohabitent dans de nombreux domaines (école, milieu professionnel, média, familles), la langue de scolarisation n'est certainement pas partagée; et c'est bien ce "non-partage » qui est l'une des sources puissantes des inégalités qui se créent dès l'école maternelle et qui se creusent tout au long de la scolarité. (Fillol, 2016a : 192)

\section{Représentations et attitudes : les dimensions identitaires derrière les usages}

Plusieurs recherches d'orientation sociolinguistique sur le terrain calédonien se sont focalisées sur les usages et surtout les représentations ${ }^{8}$ sociolinguistiques des langues dans une perspective écologique et des recherches qualitatives ont tenté d'interroger les représentations sociolinguistiques des élèves (Colombel, 2012 ; Razafi \& Favard, 2018) ou celles des étudiants et enseignants (Fillol, 2016b, 2000). L'objectif est notamment d'analyser les représentations sociales des langues et des pratiques plurilingues afin de mettre à jour les conditions dans

\footnotetext{
${ }^{7}$ Depuis 2018, les programmes locaux séparent l'enseignement des langues kanak de l'enseignement de la culture. Tous les élèves de Nouvelle-Calédonie doivent bénéficier d'un enseignement des éléments fondamentaux de la culture kanak (EFCK), de la maternelle au lycée. Et les élèves dont les parents en ont émis le vœu peuvent bénéficier d'un enseignement d'une langue kanak (et non en langue). Pour le premier degré, le volume horaire hebdomadaire est très variable, d'une demie heure à 7 heures par semaine en maternelle.

${ }^{8}$ La définition proposée par Calvet semble assez proche de la définition stabilisée proposée par les travaux en psychologie sociale au sens où les représentations assurent des fonctions de savoirs élaborés et d'orientation des comportements : «du côté des représentations se trouve la façon dont les locuteurs pensent les usages, comment ils se situent par rapport aux autres langues en présence » (Calvet, $1998: 17$ ).
} 
lesquelles les langues se vivent en milieu urbain (Barnèche, 2005 ; Colombel-Teuira et al., 2016) et en milieu scolaire (Fillol \& Vernaudon, 2004 ; Colombel, 2012, Fillol \& Colombel, 2014 ; RAZAFI \& Favard, 2018).

Plusieurs variétés de français cohabitent. Le français « kaya », « kayafou », « mélangé », tel qu'il est désigné par de nombreux acteurs, n'est pas cantonné aux quartiers urbains de la périphérie de Nouméa et est très largement plébiscité ou revendiqué pour remplir d'autres fonctions ; en témoigne cet extrait d'autobiographie langagière :

En parallèle du français, j'ai aussi appris à développer le « français kaya ». J'ai grandi et j'ai été à l'école à Rivière-Salée. Dans les quartiers, le « français kaya » est très utilisé. Je définirai le « français kaya » par une forme de français revisitée, dans un langage familier et propre à la Nouvelle-Calédonie. C'est parler le français de façon «débarrassée » et avec des expressions faisant référence à des éléments de la vie de tous les jours («tu fous la flemme », « kayafou », « tal toul », « le truc à lui là », etc.). C'est comme si c'était une façon volontaire de parler le français n'importe comment, en ne respectant pas la grammaire.

Je suppose qu'une fois de plus, le «français kaya » est un moyen de se différencier du français soutenu et des français de France. Je pense qu'il y a une volonté de ne pas être associé aux «blancs » pour les kanak et personnes d'autres ethnies, mais aussi de ne pas être associé aux français de France pour les calédoniens ou caldoches. (...).

Ce français est beaucoup utilisé dans les quartiers mais est également présent sur tout le territoire. J'ai donc acquis naturellement et sans m'en rendre compte cette variation du français. Selon le contexte ou les personnes à qui je m'adresse, je « jongle » entre le français et le « français kaya ». (Autobiographie langagière $n^{\circ} 162$, corpus V. Fillol)

La variation apparait alors non pas comme une simple conséquence de la différenciation sociale, mais comme un agent actif des antagonismes sociaux et/ou ethniques ${ }^{9}$.

Une autre catégorisation est souvent utilisée pour référer à une pratique du français qui n'est pas celle des «zoreilles ${ }^{10}$ », ou des détenteurs de la langue légitime; celle de français calédonien :

Le français calédonien est pour moi une façon de se différencier du français standard. En effet, je ne parle pas de la même façon, en utilisant des expressions propres de la Nouvelle-Calédonie. Pour moi, le français calédonien est une langue identitaire. Étant une petite métisse du pays, appartenir à deux ethnies différentes, deux cultures différentes, je ne peux me permettre de choisir entre les deux. Alors pour moi, le français calédonien me permet de m'identifier comme étant une Calédonienne. (A., Autobiographie langagière $n^{\circ} 29$, corpus V. Fillol).

Dans un texte qui accompagne une carte mentale, un étudiant présente son bilinguisme français-nengone comme le pilier «de son identité linguistique et culturelle »; cependant il souligne la « complexité de la langue dans ( $\mathrm{sa}$ ) scolarité » et marque une distinction importante entre le « français du quotidien et le français académique ».

\footnotetext{
${ }^{9}$ La répartition ethnique, héritée de la période coloniale, est toujours un principe organisateur de la société calédonienne (Colombel, 2012).

${ }^{10}$ Désigne les métropolitains.
} 


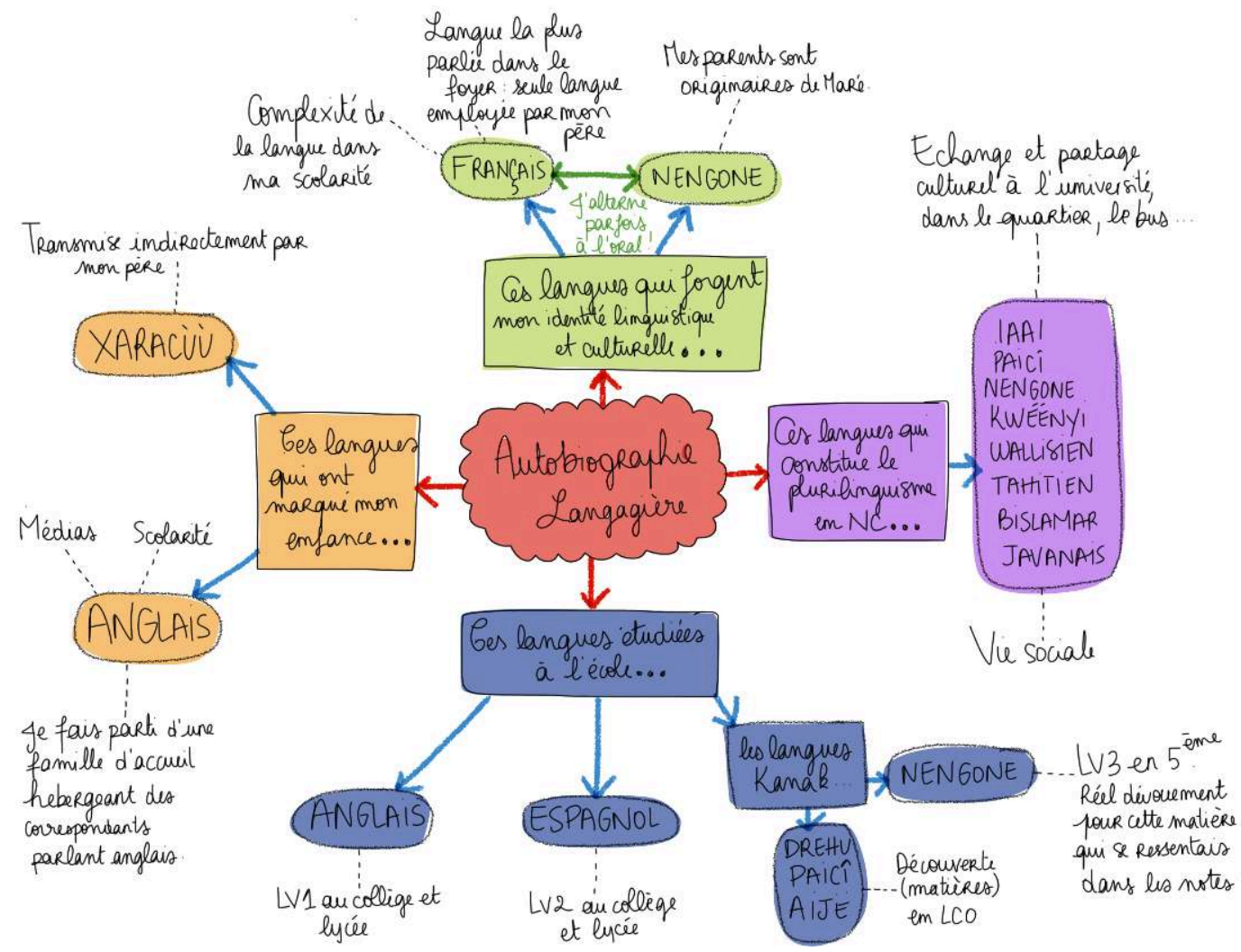

On me dit souvent que ma maitrise de la langue française laisse à désirer : « Tu as un français trop calédonien » ou encore « Ton français n'est pas correct ». Voici quelques exemples de microagressions dont j'ai été victime. C'est pour cela que mon rapport avec le français scolaire (ou français «normal ») reste délicat. J'ai toujours eu un complexe vis-à-vis de cette variante de la langue française. (...)

Dans ma vie de tous les jours, c'est le langage populaire et familier qui prévaut sur le langage académique. (W., Autobiographie langagière $n^{\circ} 141$, corpus V. Fillol)

Comme tous les Calédoniens, les différents acteurs de l'école (élèves comme enseignants) ont des répertoires langagiers pluriels et entretiennent parfois des rapports complexes, voire compliqués avec leurs différentes langues ou usages de ces langues.

\section{Quels outils de compréhension des usages langagiers et de l'agir enseignant ?}

Articulant recherche et formation d'une part et interdisciplinarité d'autre part (linguistique océanienne, sociolinguistique et sociodidactique), les enquêtes, observations et analyses de micro-contextes $^{11}$ ont permis l'élaboration d'outils visant à donner des repères (socio)linguistiques aux enseignants, à faire évoluer sensiblement les représentations et surtout contribuer à une meilleure compréhension des productions langagières des élèves.

\footnotetext{
${ }^{11}$ Dans le cadre du programme de recherche ANR École Plurilingue Outremer (ECOLPOM), nous avons effectué des centaines d'heures d'observation de classe dans les 3 Provinces (Sud, Nord et Iles Loyautés) pour identifier les modalités d'utilisation des langues et les pistes sociodidactiques envisageables (enseignement bilingue, coenseignement, éveil aux langues...). (Fillol \& Colombel, 2014)
} 


\section{La grammaire comparée : des savoirs linguistiques pour changer de regard sur les usages}

L'un des repères que nous proposons - notamment en formation continue - concerne des éléments théoriques de grammaire comparée ${ }^{12}$ entre les langues océaniennes et le français en nous focalisant sur la grammaticalisation (les types de phrase, l'ordre des mots dans la phrase, la négation, le genre, le nombre, les déclinaisons, la polyfonctionnalité ${ }^{13} \ldots$ ) et la mise en discours.

L'analyse de productions linguistiques et discursives à l'oral et à l'écrit d'élèves ou d'étudiants plurilingues vise à envisager l'erreur dans la langue « cible » comme révélateur de compétences dans la langue/variété source :

98 Vani : moi moi fin l'école

99 Laura : répète moi ça/

100 Vani : moi fini l'école et mon frère bé (.) bé ma mère il s'est trompé de jour Pendant les vacances (extrait) calque syntaxique (Païta, 2012), Corpus ${ }^{14}$ Colombel, 2012

Vani entend au quotidien deux langues polynésiennes (le wallisien et le tahitien). Dans cet extrait, elle calque la structure syntaxique de sa «première » langue : le wallisien, une langue isolante ; on observe l'emploi d'un pronom à la $1^{\text {ère }}$ personne, invariable « moi »; « fin » qui a la fonction de prédicat (polyfonctionnalité des mots en wallisien) et l'objet.

Comme «ça ne marche pas » en français, l'enseignante lui demande de reformuler. Vani change fin en fini; elle ajoute une marque flexionnelle (conjugaison) qui correspond au fonctionnement du français. Même si la formulation du tour 100 ne correspond pas encore à la structure attendue en français ${ }^{15}$, notons qu'à l'invitation de la maitresse, Vani est capable d'intégrer des corrections qui correspondent bien au fonctionnement du français. D'une part, nous formulons l'hypothèse que si elle bénéficiait d'un enseignement lui permettant de structurer les usages dans les deux langues (comparaison, manipulation... ) elle arriverait avec plus d'efficacité à produire des énoncés « attendus » en français et d'autre part nous soulignons qu'elle témoigne de compétences qui peuvent servir de point de départ à un enseignement pluriel structuré (partant des compétences épilinguistiques vers un travail métalinguistique et non pas simplement la production de reformulations « imposées » par la maitresse).

Au-delà des considérations sur le fonctionnement morphosyntaxique des langues en présence, l'entrée par la grammaire comparée permet d'élargir la réflexion sur les usages langagiers, tant avec les élèves qu'avec les enseignants en formation (initiale ou continue). Très souvent, les interférences liées aux langues océaniennes (même à l'état de substrat) ou les usages oraux sont évalués négativement car jugés trop distants de la « vraie grammaire » ou de la « vraie langue » française, ils peuvent néanmoins servir de marchepied pour découvrir et manipuler la notion d'usage. Dans l'extrait $2^{16}$, on observe l'emploi d'un pronom personnel (dans une forme invariable) par un enfant, puis au tour 11, cet emploi est repris par la mère.

\footnotetext{
${ }^{12}$ Champ d'études développé par Vernaudon (2009) et Colombel-Teuira.

${ }^{13} \mathrm{La}$ polyfonctionnalité ou encore la transcatégorialité sont selon S. Robert « un moyen d'optimisation des systèmes linguistiques, permettant à un minimum de formes d'avoir un maximum de fonctions » (2003 : 18). Dans de nombreuses langues océaniennes, un substantif, par exemple, peut endosser la fonction de sujet, d'objet, de prédicat ou encore d'épithète selon le contexte d'apparition.

${ }^{14}$ Les prénoms attribués sont des pseudonymes, à l'exception de Claire (infra), co-auteure de cet article. Il s'agit d'un extrait de la transcription d'une captation faite en section des grands (Vani est une élève de 5 ans).

${ }^{15} \mathrm{La}$ formulation du tour 100 est un calque syntaxique du wallisien (ainsi que de différentes langues de la région) qui semble se sédimenter dans les usages du français en Nouvelle-Calédonie.

${ }^{16}$ En formation, nous utilisons régulièrement des enregistrements (audio, vidéo), des copies d'élèves ou des transcriptions d'interactions. Dans un premier temps, l'irruption d'un français « illégitime » dans un espace de formation provoque des rires. Cependant, ce « français cassé », selon une autre expression locale, correspond très souvent aux usages quotidiens réels des enseignants.
} 
Cette tournure « orale » est répandue dans les usages calédoniens (« fais montrer à moi », «j'ai vu à toi »). Dans un contexte de formation, ce simple exemple permet de questionner les usages langagiers (réels) des enseignants ainsi que leur(s) rapport(s) aux différentes variétés de français (ou d'autres langues) dont ils usent.
7. Mère c'est pas euh, pas trop court
8. Enfant t'as dit à moi maman/
9. Mère eu:: einhh/
10. Enfant t'as dit à moi
11. Mère non j'ai pas dit à toi
12. Enfant gad'

Au magasin (extrait), français de la maison (Nouméa, centre ville), corpus Colombel-Teuira, 2016

Cependant, si ces outils - de grammaire comparée - permettent de faire évoluer une posture évaluative ou "normative » vers une posture plus compréhensive, ils peuvent accroitre la tendance à focaliser l'attention et l'analyse des productions langagières sous le seul angle des compétences linguistiques ${ }^{17}$ (phonologiques, orthographiques, morpho-syntaxiques), qui est (déjà) ancrée dans les habitus et même l'identité professionnelle de nombreux enseignants du premier degré (Colombel, 2012 ; 2014).

\section{Éveil aux langues océaniennes : légitimer les langues dans la classe et développer les compétences métalinguistiques}

Un autre repère ou outil relève de l'éveil aux langues océaniennes - développé dans plusieurs pays et territoires d'outre-mer tant dans les formations d'enseignants que dans les salles de classe. Dans le contexte néo-calédonien, cette approche didactique se développe en s'ouvrant à la variation, à l'instar des travaux suisses $\left(\mathrm{EOLE}^{18}\right)$, et à la diversité culturelle.

Du point de vue de la formation des enseignants, l'éveil à la diversité agit sur 1) les représentations sur les langues océaniennes en les modifiant (un peu) notamment sur la perception de la grammaticalité des langues (en se rendant compte que la «grammaire du français » n'est pas la seule grammaire du monde) et sur 2) les conceptions de l'apprentissage ou du développement des compétences langagières, repositionnant modestement le curseur d'évaluation de la «maitrise de la langue française ».

Les apprenants construisent ainsi une nouvelle relation aux savoirs scolaires, affranchie des conceptions (normatives, voire prescriptives) de la culture dominante (Candelier, $2003: 45$ ). Se développent ainsi : l'aptitude à observer ${ }^{19}$, comparer et analyser les langues; l'intérêt et l'ouverture vis-à-vis de la pluralité linguistique, la légitimation des identités ou de certaines pratiques plurilingues dans la classe. Claire Colombel-Teuira, qui a produit de nombreux outils pour la classe, a observé - pour certains élèves, même très jeunes - que la légitimation de leur déjà-là (linguistique, langagier et culturel) pouvait avoir une incidence sur l'estime d'eux-

\footnotetext{
${ }^{17}$ Certaines ressources pédagogiques mises à disposition par la Direction de l'Enseignement de la Nouvelle Calédonie (DENC) sont susceptibles de renforcer les représentations amalgamant langagier et linguistique. À titre d'exemple, dans un document intitulé «enseigner le vocabulaire », la partie détaillant les objectifs liés à l'enseignement du vocabulaire pose comme préalable : «Toute séance doit comporter 2 objectifs langagiers : un d'ordre lexical et un autre d'ordre syntaxique ». Document DENC, consulté en ligne :

https://denc.gouv.nc/sites/default/files/documents/enseigner_le_vocabulaire_au_cycle_1_document_daccompag nement.pdf

${ }_{18} \mathrm{https}: / / \mathrm{www} . i r d p . c h /$ institut/eole-education-ouverture-langues-ecole-1569.html

${ }^{19}$ L'ORL (Observation réfléchie de la langue) dans les programmes français 2002 (= programmes calédoniens 2006) permettait de légitimer institutionnellement cette démarche pédagogique, d'inspiration socio-constructiviste en transformant l'ORL en observation réfléchie et comparée du français et des langues locales.
} 
mêmes (représentation dite) et leur attitude en classe (représentation agie) (Colombel-Teuira, 2019).

Une recherche collaborative récente dans une école primaire d'un quartier défavorisé de Nouméa montre que l'éveil aux langues peut participer au développement des compétences métalinguistiques et aide même les élèves à conceptualiser le principe alphabétique. Enfin, la visibilisation et la sollicitation des compétences plurilingues des élèves en classe peut participer à développer un regard positif des enseignants sur leurs élèves (Colombel-Teuira, Fillol \& Bousquet, 2020).

\section{Autobiographie langagière et autoscopie : des dispositifs réflexifs}

À côté de ces « outils » contextualisés, co-construits et proposés avec des enseignants, nous avons aussi développé des dispositifs que nous qualifierons de réflexifs pour accompagner les (futurs) enseignants dans le changement de représentations, d'attitudes, la conscientisation, l'appropriation de concepts : l'autobiographie langagière et l'autoscopie. Concilier conception de l'enseignement/apprentissage et conception de ce qui fait " langue » conduit à prendre en compte le déjà-là des élèves (ou celui des enseignants) (ibid.) :

Depuis le virage socio-constructiviste dans les théories de l'apprentissage et l'avènement des approches communicationnelles, le " sujet apprenant 》 est devenu la pierre angulaire des démarches didactiques. (Razafi \& Favard, 2018 : 318)

Le travail autour des biographies ${ }^{20}$ permet d'aborder avec les étudiants et enseignants (en formation continue) des questions et problématiques sociolinguistiques et sociodidactiques contextualisées : conscientisation des plurilinguismes ; transmission ou rupture de transmission des langues familiales; statuts et fonctions assignées aux langues; poids des attitudes et discours normatifs ; discriminations, assignations identitaires... (Fillol, 2016b et 2020). En témoigne l'extrait du portfolio d'une enseignante en formation continue :

La posture réflexive, que la formation du DU FLES, option interculturalité ${ }^{21} m$ 'a demandée de développer, $m$ 'a replongée dans des souvenirs d'enfance. (...) Dans la cour de l'école, je ne parlais pas "la langue des Blancs », " je ne phrasais pas », comme on dit en NC. Aujourd'hui, je suis plutôt fière de me dire que j'avais des compétences polynomiques, cependant à l'époque, maitresse Mathilde, une française de "Frônce 22 " me signifiait mes "lacunes » en me mettant toujours au coin parce que je ne savais toujours pas conjuguer le verbe à l'imparfait... Mais, au fait c'est quoi "un-parfait ». Ce n'est qu'une anecdote parmi tant d'autres. Je suis aujourd'hui particulièrement vigilante avec mes élèves pour lever toutes sortes

\footnotetext{
${ }^{20}$ Dans le cadre d'un enseignement intitulé Approches plurielles en didactique des langues-cultures, situé au dernier semestre de la formation, les étudiants de Licence Lettres et Licence Langues et cultures océaniennes rédigent leur autobiographie langagière sur une période de 10 à 12 semaines et sont invités à utiliser carte mentale, schéma ou dessin. Cet écrit réflexif est aussi demandé aux enseignants en formation continue (DU Fles et DU LCOA : Langues et cultures océaniennes et Apprentissages) et intégré à un portfolio. Ce corpus est à ce jour constitué de plus de 200 textes, recueillis entre 2015 et 2020.

21 Le Diplôme d'Université Français Langue Étrangère et Seconde «Océanie » (DU FLES) a pour but d'accompagner les professionnels de l'enseignement des langues et plus largement de l'éducation, vers une gestion intégrée et contextualisée des pluralités sociales (linguistiques, culturelles). Cela suppose de se construire des repères pluridisciplinaires mais aussi de pouvoir effectuer un retour analytique et critique sur ses propres pratiques professionnelles, d'où la place centrale accordée aux approches réflexives au sein de cette formation (Fillol, Geneix, Razafimandimbimanana, 2018).

22 Référence ici à la prononciation locale qui est régulièrement sujet de plaisanterie, d'imitation... par les calédoniens eux-mêmes et écrit comme tel dans les bandes dessinées de Bernard Berger (La brousse en folie). Note des auteurs.
} 
de malentendus (linguistique ou didactique). Je ne punis pas les enfants pour des erreurs de conjugaison ou de syntaxe, mais ces quiproquos me font parfois sourire.

(...) Les apports en sociolinguistique lors de la formation au DU, ont particulièrement fait écho à mon parcours. En effet, les travaux en sociolinguistique (Ledegen, Léglise) décrivent la norme comme une réalité plurielle qui est régie surtout par des forces sociales. En d'autres termes "c'est la communauté linguistique qui attribue des valeurs extralinguistiques à la variation linguistique et elle sanctionne ainsi certains emplois langagiers ». Dès lors, cette norme peut engendrer une insécurité linguistique : certains locuteurs se sentent incapables de maitriser la norme légitime, ou ne souhaitent pas l'apprendre car cela implique de renier les pratiques de sa communauté. (Bousquet, 2018 : 12)

Enfin, avec les enseignants en poste, nous proposons l'autoscopie, un outil d'analyse de leurs pratiques. L'autoscopie est une pratique répandue dans les formations professionnelles (Beckers et Leroy, 2011). Il s'agit de permettre aux enseignants d'observer leurs pratiques professionnelles à travers des captations vidéo. Dans le cadre des projets accompagnés, pour les visionnages, nous avons procédé selon le souhait des enseignants ; soit ils regardaient une première fois les prises de vue seuls, soit nous les visionnions ensemble. Les analyses compréhensions s'élaborent dans la négociation, en veillant à ne pas tomber dans le travers de la justification comme a pu le montrer Olivesi (2012) dans le cadre de la formation syndicale. L'intérêt du visionnage commun réside dans le dialogue réflexif qui nait de la «confrontation » des regards.

\section{Un exemple d'approche polynomique}

Nous faisons nôtres plusieurs principes sociodidactiques pour des recherches à visée interventionniste et avons, en ce sens, collaboré à différents projets dont l'origine était souvent en lien avec des questionnements des enseignants ${ }^{23}$ (ou acteurs de l'école). Si les quelques outils présentés supra participent à la conscientisation des enseignants (et des élèves) de la complexité et la richesse des répertoires (linguistiques et langagiers) de chacun, le "saut» didactique n'est pas moins compliqué. Nous proposons de détailler quelques aspects didactiques d'un enseignement basé sur le déjà-là pluriel des élèves comme une ressource ; les exemples et illustrations présentés sont essentiellement tirés d'un travail collaboratif, de près de sept ans, avec une enseignante experte ${ }^{24}$.

\section{Des usages sociolangagiers quotidiens aux usages scolaires}

Vahimiti Bousquet (désormais VB) exerce en CP (élèves de 6 ans), dans une école située dans un quartier populaire (en zone d'éducation prioritaire). Les élèves sont pour la grande

23 La diffusion de recherches co-élaborées avec des enseignants semblent plus «concernantes » pour les enseignants en formation initiale et continue (Colombel-Teuira et Fillol, 2020).

${ }^{24}$ Vahimiti Bousquet est institutrice et a exercé essentiellement dans un quartier populaire de Nouméa. Après avoir débuté sa carrière au Cours Moyen 2 (élèves de 10 ans), elle a choisi d'enseigner en Cours Préparatoire (élèves de 6 ans). Elle a longtemps été maitresse d'accueil pour de futurs enseignants et est maitre formatrice depuis 2019 . Il faut souligner qu'elle a personnellement subi l'intolérance, voire la maltraitance linguistique quand elle était élève. Bien qu'elle soit consciente des répercutions psychoaffectives et identitaires, ainsi qu'en matière de carrière scolaire, de l'imposition d'une mono norme à l'école, une partie de notre collaboration a consisté à déconstruire un paradoxe ressenti : d'une part mettre l'élève au cœur des apprentissages, c'est-à-dire le considérer pour ce qu'il est, et d'autre part répondre aux injonctions de «maitrise » de la langue française des programmes (avec des attendus de fin de cycle) qui sont amplifiées par diverses formes de pression sociale (hiérarchie, mise en concurrence entre collègues...). 
majorité des enfants océaniens au contact de plusieurs langues, vivant et pratiquant au quotidien différentes cultures mais ne maitrisant pas tous les codes scolaires - ou pour reprendre les termes de Bourdieu n'ayant pas le capital culturel. Cette enseignante a fait un pari audacieux sur les compétences de ses élèves, pourtant réputés en difficulté. Son pari est de valoriser les langues-cultures en présence - le capital des élèves - pour faire cheminer ces enfants vers les compétences scolaires (programmes, socle commun de connaissances, de compétences et de valeurs) et ce dans toutes les disciplines, y compris la «maitrise de la langue ». Le schéma cidessous représente le cheminement que VB propose à ses élèves.

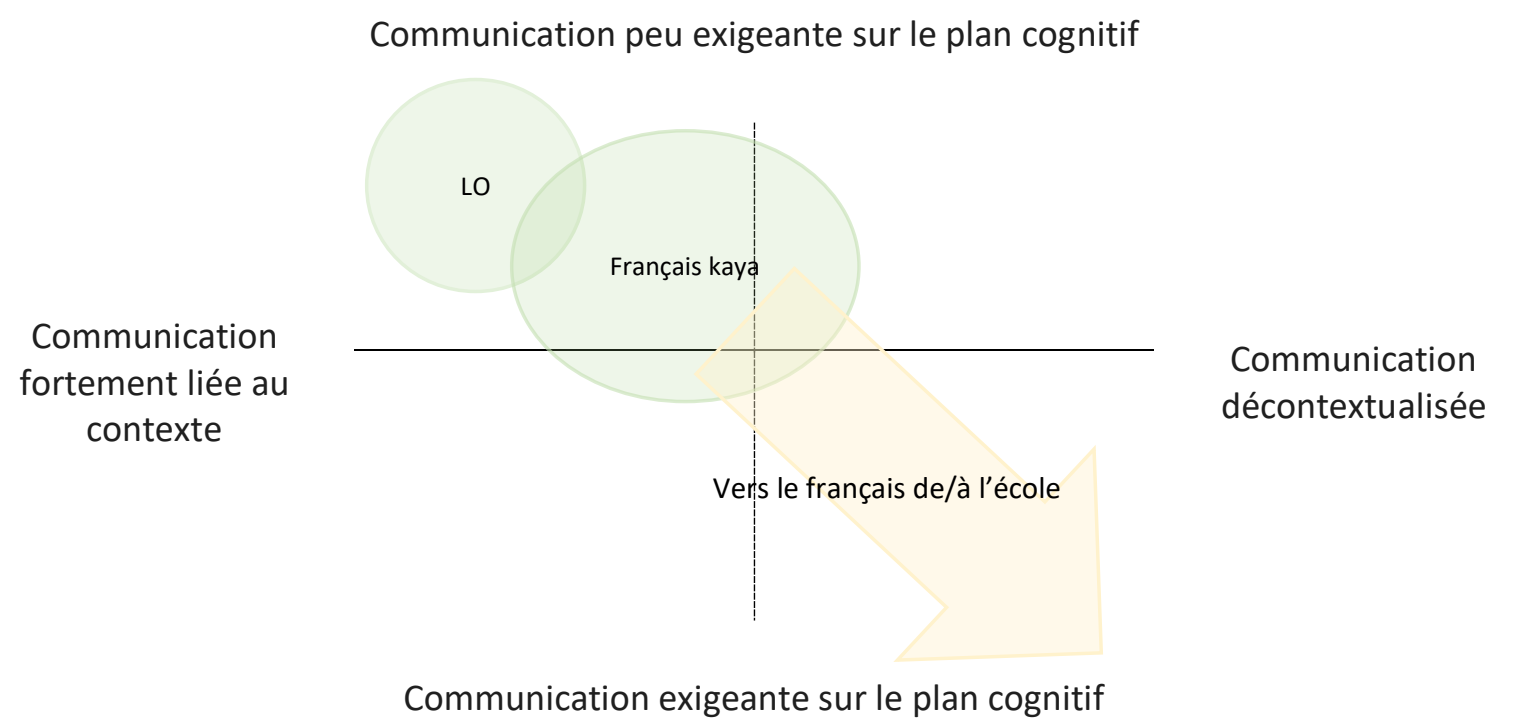

Schéma 1 : Positionnement des élèves, d'après la grille de Cummins (1983)

Une des premières implications didactiques est un travail de contextualisation pensé dès la conception des outils du maitre et perpétuellement réajusté jusque dans les phases de bilan de la mise en œuvre - l'enseignante veille constamment à ce que son attitude bienveillante n'altère pas la perception des élèves de la situation didactique, i.e. des attendus langagiers, cognitifs, posturaux... À partir d'une observation fine des élèves, il s'agit d'identifier ce qui dans leur répertoire social (leur déjà-là) constitue une entrée potentielle dans de nouveaux apprentissages. Les phases de mise en projet, mise en situation (construction d'un « conflit sociocognitif ») sont alors extrêmement dépendantes du contexte, de la situation et de la maitrise du geste professionnel de tissage ${ }^{25}$ de l'enseignant.

Notre collaboration avec VB a commencé avec différentes séquences d'éveil à la diversité linguistique, qui nous ont permis de constater que l'implication des parents ainsi que l'appétence des élèves pour l'école prenaient une nouvelle dimension, dans un temps assez court. En effet, au-delà de procurer un sentiment d'être « enfin » légitime, la mise en œuvre de l'éveil à la diversité (linguistique et culturelle) a permis aux parents de prendre pleinement leur rôle d'acteur de et dans l'école et aux élèves d'exprimer leur curiosité et de développer des compétences métalangagières (Colombel-Teuira, 2019). De ces constats encourageants, sont nés différents projets prenant appui sur et valorisant les répertoires tant langagiers que

\footnotetext{
${ }^{25}$ « La métaphore renvoie à l'idée que le savoir se construit et prend sens d'abord dans le « déjà là ». Les gestes de tissage traduisent le souci chez l'enseignant de relier l'avant et l'après de la tâche, le dedans et le dehors de la classe, permettant de faire du lien avec ce qui a été appris à l'école ou ailleurs, dans les leçons ou travaux précédents, dans l'expérience personnelle, les lectures. Ces gestes de tissage sont essentiels pour les élèves " décrocheurs », ou «suiveurs passifs » qui «font » consciencieusement les tâches sans en comprendre les finalités ». (Morel et al., $2015: 68$ ).
} 
linguistiques des élèves. C'est essentiellement une démarche herméneutique qui a conduit cette enseignante à réfléchir à la didactisation de la variation.

Le concept sociolinguistique de polynomie développé par Marcellesi (1983) s'applique à des langues ${ }^{26}$ comme le corse qui aujourd'hui est assez bien documenté (Marcellesi, 1983 ; Sorba, 2016). La transposition sociodidactique de ce concept s'est révélée opérante pour la didactique des langues minoritaires et l'éducation au plurilinguisme (Cortier et Di Meglio, 2007).

Dans le contexte néo-calédonien, ni le français ni les langues océaniennes ne peuvent être qualifiées de langue polynomique. Cependant une approche reconnaissant et valorisant la variation est possible à la condition d'effectuer un travail de conscientisation de l'existence de normes différentes d'une part, et d'autre part, un travail sur les représentations des enseignants sur la légitimité des différentes variétés (d'usages) des langues. Ce changement de paradigme ${ }^{27}$ est donc une construction pensée.

\section{Quand la parole se libère}

Dans l'approche polynomique de cette enseignante, la manipulation du langage en classe repose sur l'aménagement d'espaces (discursifs) que les élèves co-construisent. Ces espaces ont un effet « libérateur » sur les élèves qui prennent plus facilement la parole et manifestent leur curiosité. La fonction heuristique du langage, outil de développement des compétences épimétalangagières est privilégiée. Enfin, ces espaces offrent les conditions d'élaboration et de mobilisation des connaissances, compétences ou attitudes. En d'autres termes, libérer la parole concerne tant un aspect conatif que cognitif, deux aspects intiment liés.

VB légitime les répertoires de ses élèves en accueillant leurs usages langagiers dans des pratiques discursives scolaires. Les premiers effets que nous pouvons observer sont d'ordre conatif. Dans les 2 extraits suivants, l'enseignante laisse les élèves s'exprimer « comme ils le souhaitent » lors d'un débat philosophique sur l'école. Ainsi, accepter le(s) code(s) de chacun permet de cibler un objectif langagier - et non plus seulement linguistique, mais surtout de valoriser ou légitimer la parole de chacun.

1 Enseignante Alors/pourquoi tu as choisi cette image Albert/

2 Albert Beh par que j'aime bien les copè

3 Enseignante Pace que tu aimes bien tes/

4 Albert Copè

5 Enseignante Tu aimes bien tes copains $\backslash$ (2) euh mais moi je trouve ça extraordinaire $\backslash$ Albert/ est-ce que tu as autre chose à dire/

6 Albert Euh :: (2) euh :: c'est pour (3) non (1) bon〉

7 Enseignante Et ben/on peut l'applaudir (applaudissements des élèves) il vient à l'école parce qu'il aime bien ses copains

Débat philosophique (extrait)- l'école (CP, Nouméa, ZEP), corpus Colombel-Teuira, 2018

\footnotetext{
${ }^{26}$ « Langues dont l'unité est abstraite et résulte d'un mouvement dialectique et non de simple ossification d'une norme unique, et dont l'existence est fondée sur la décision massive de ceux qui la parlent de lui donner un nom particulier et de la déclarer autonome des autres langues reconnues » (Marcellesi, 1983 : 314 cité par Blanchet, 2016:91).

${ }^{27}$ Partant du constat que dans l'institution scolaire les élèves «subissent une intolérance forte à toute variation linguistique (celle du français) et une tolérance, également forte, à la variation linguistique (celle du corse) » (2016 : 103), Sorba souligne la complexité et la difficulté à appliquer le concept de langues polynomiques dans « un lieu marqué par un système très éloigné du concept » (p. 106).
} 
Dans le cas d'Albert qui ne parle que très peu et répond souvent par des mimiques faciales, il s'agit de ne pas le reprendre explicitement mais de proposer une " version » plus académique (tours 5 et 7), ici essentiellement sur des points phonétiques ${ }^{28}$ ( ${ }^{*}$ copè, pour copain) et l'emploi d'adjectifs possessifs ${ }^{29}$. Pour libérer la parole de ce « petit parleur», VB choisit de remettre à plus tard le travail de structuration (phonétique et syntaxique).

Pour Mary, qui est originaire de Fidji, le bau ${ }^{30}$ et l'anglais sont clairement des langues identitaires qu'elle pratique à la maison, et le français représente pour elle la langue de l'école (de l'extérieur). Cette élève qui en début d'année refusait de prendre la parole, accepte, dans l'exemple ci-dessous, de participer quand on lui propose de commencer en anglais. Petit à petit, ses prises de parole se sont étoffées.

$(\ldots)$

8 Enseignante Est-ce que quelqu'un veut partager quelque chose/ (2) sur son image (1) euh Mary/ come on

9 Loredys

Allez/ come on (1) Allez/ come on

10 Enseignante

Oh you want to tell in English/you want to speak in English/or in French

11 Mary

In English

12 Enseignante

after you translate it

13 Mary

Euh $\mathrm{mm}$

14 Enseignante

Ok/ thanks/

15 Mary

Me I take this photo because euh if I:: XXX me I will do this and I will go the mountain (2) after if I ha-if I have some (2) some children I will go there in the mountain and after I will see this (2) like school there

16 Enseignante Okvery good

17 Kilian En français::/

18 Enseignante En français

19 Sinöe Ça veut dire quoi/

20 Mary Ben moi si j'étais (1) et euh grande et si je eh je

21 Loredys Ben tu peux regarder (montre à Mary son image)

Débat philosophique (extrait)- l'école (CP, Nouméa, ZEP), corpus Colombel-Teuira, 2018

Les aspects cognitifs n'en sont pas pour autant mis de côté. La conscientisation des différentes normes de la langue française (et plus largement d'usages) s'est peu à peu construite à partir d'activités routinisées pour permettre aux élèves d'automatiser l'emploi de nouveaux usages. Traditionnellement, les rituels permettent de libérer une partie de la charge cognitive favorisant ainsi les apprentissages - en repoussant la $Z P D^{31}$. VB a proposé à ses élèves un rituel « du français de la maison au français de l'école » où l'usage familial (familier) côtoie au quotidien les usages rencontrés à l'école ${ }^{32}$ de façon normale. Dans un premier temps, le rituel était proposé dans un espace de la classe identifié (le coin bibliothèque) et se déroulait soit en début de matinée ou au retour de récréation. Cet espace symbolique de «jeu avec la langue»

\footnotetext{
${ }^{28}$ Albert est originaire Lifou (Loyauté). En drehu, langue de ses parents, il n'existe pas de voyelle nasale. Dans

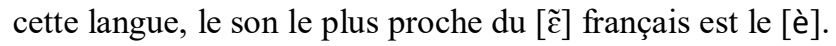

${ }_{29}$ Dans les usages ordinaires du français calédonien, le possessif s'exprime plutôt avec une construction prépositionnelle (les copains à moi).

${ }^{30}$ Langue mélanésienne majoritaire des iles Fidji, aussi appelé bauan (qui vient de l'ile de Bau).

${ }^{31}$ Zone Proximale de Développement.

${ }^{32}$ Usage que les élèves appellent le «parler des Blancs ».
} 
servait à l'enseignante à faire la transition vers la classe et les attendus scolaires. L'enseignante proposait aux élèves de réfléchir à ce qu'on dit et comment on le dit. Elle proposait un énoncé aux élèves en «français de la maison » et les invitait à proposer des formulations en « français de l'école» ou inversement. Par exemple, les élèves sont invités à décortiquer l'énoncé « maitresse, c'est bon si tu dis à lui d'arrêter de faire mauvais à moi ${ }^{33}$ » pour proposer un énoncé « qu'on pourrait écrire »; ou comme dans l'extrait suivant, les élèves sont invités trouver un équivalent en français de la maison.

6 Claire je suis trop heureuse d'aller à la plage ce weekend

7 Enseignante vous diriez comme ça vous/ comment on dirait (.) nous à la maison/

8 Kilian mais en fait, mais t'as PEUR/

9 Loredys mais c'est : parce qu'y a des requins/

10 Enseignante maitresse Claire (1) tu as peur/ moi, j'ai pas compris çal

11 Claire MAIS non/ (.) pas du tout/ je n'ai pas peur (1) au contrai :re/ (surjoue l'intonation) je suis trop heureuse d'aller à la plage ce weekend +

12 Loredys mai ::s (1) on dirait que t'es contente

13 Claire ben/oui (.) j'aime beaucoup aller à la plage

14 Albert mais pourquoi t'as dit que t'es peureuse/

15 Kilian tu : peux dire un coup encore/

16 Claire (surjoue l'intonation) je suis trop heureuse d'aller à la plage ce weekend+

17 Albert c'est comme si tu dis t'es heureuse

18 Moeata mais moi j'entends peureuse

19 Enseignante peut-être qu'elle a dit heureuse/ (2) mais alors/ (.) qu'est-ce qu'elle a dit (.) avant heureusel

20 Loredys je suis trop heureuse

21 Kilian elle a dit trop

22 Enseignante (montre les mot outils de la classe)

23 Albert c'est trop

24 Kilian ah :: TROP/ (à Claire) mais le -p- on le dit pas

Rituel langue de l'école (extrait) - liaison - (CP, Nouméa, ZEP), corpus Colombel-Teuira, 2018

Ce rituel, que les élèves prenaient comme jeu de traduction, a peu à peu pris une nouvelle dimension didactique. Dans l'extrait ci-dessus, par exemple, les élèves "découvrent» les liaisons facultatives en français et aiguisent leur capacité à prendre des indices; Loredys (tour 12) relève que Claire semble « contente » ce qui permet à Albert (tour 17) d'identifier le lexème « heureuse » qui lui parait plus cohérent. Ainsi après avoir vérifié et validé le sens de la phrase proposée par Claire, les élèves ont cherché comment ils «traduiraient » cet énoncé à leurs cousins ou leurs copains ${ }^{34}$.

Si au début, l'impulsion venait systématiquement de l'enseignante, les élèves ont manifesté leur curiosité notamment en proposant des expressions qu'ils voulaient apprendre à dire « en français de l'école ». Quand Uriel demande à VB « Maitresse, comment on dit en français de l'école, je démarre avec la bande ! », pour prévenir le directeur qu'il quittera l'école avec son

\footnotetext{
${ }^{33}$ On pourrait reformuler : « Maitresse, j'aimerais que tu lui dises d'arrêter de m'embêter ».

${ }^{34}$ Leur proposition finale : «c'est fin choc aller baigner la mer ».
} 
grand frère et des copains du quartier, il semble réinvestir la répartition fonctionnelle des usages.

Petit à petit, les élèves ont utilisé les stratégies apprises (développées) grâce au rituel pour vérifier s'ils comprenaient ce qu'ils lisaient ou ce qui était dit en classe. À partir de ces « tâtonnements d'interprétation » des élèves, l'enseignante les a peu à peu guidés vers la prise d'indices, notamment en leur faisant verbaliser leurs stratégies inférentielles ${ }^{35}$ ( « mais comment tu sais ? " "pourquoi tu dis... ? »). La stratégie didactique de l'enseignante est là encore de partir de ce que les élèves sont capables de produire. Et par un jeu de structuration langagière et de la pensée, dans l'interaction verbale, les élèves sont amenés vers une nouvelle conduite discursive; de la description d'une série d'actions à l'élaboration de stratégies/procédure complexifiant ainsi tant les contraintes linguistiques que langagières, notamment en accroissant les exigences cognitives. En d'autres termes, tout en restant dans la ZPD de ses élèves, notamment en acceptant leurs usages langagiers ordinaires, cette enseignante balise une voie vers le processus de «secondarisation » pour accompagner les élèves vers la compréhension, la conceptualisation ou pour reprendre les mots de Bautier et Goigoux pour que les élèves « s'autorise[ent] à faire circuler les savoirs et les activités d'un moment et d'un objet scolaire à un autre » $(2004: 91)$.

Pour l'enseignante, libérer la parole de ses élèves nécessite de repenser la place de son oral comme celui des élèves. D'une part, l'irruption de forme d'oral réflexif en dehors des phases de bilan (institutionnalisation, objectivation), souvent reléguées en fin de séance et parfois escamotées entraine des réajustements permanents et d'autre part l'enseignante doit sans cesse veiller à ce que la forme de liberté sociolangagière et discursive ne masquent pas les attendus des tâches cognitives en jeu.

Les quelques exemples que nous avons développés sont issus de travaux, de questionnements construits avec une enseignante particulièrement sensible à la question de la bientraitance linguistique. L'approche polynomique qu'elle a développée lui est donc personnelle. Si certains enseignants proposent à leurs élèves des rencontres-comparaisons de différentes variétés (ou niveaux de langue), ce qui est l'entrée la plus observée, peu d'entre eux accordent une place (fonctionnelle) aux usages des élèves dans la mise en œuvre de leurs enseignements.

\section{Conclusion}

Dans le contexte calédonien, nous n'avons pas encore le recul nécessaire pour évaluer (mesurer) les effets à long terme d'une approche didactique polynomique, notamment parce que ces approches relèvent quasi exclusivement des convictions et de l'usage de la liberté pédagogique des enseignants.

La question de la variation - et de son traitement didactique - est une question ancienne. Nous dirons qu'elle est en Nouvelle-Calédonie objet de débats, voire de tensions et probablement un objet didactique non suffisamment identifié. Il nous semble nécessaire de travailler avec les acteurs à la prise de conscience de la variation (et de ses fonctions ou valeurs y compris symboliques et affectives) et à la nécessité de développer des gestes d'étayage, à favoriser le passage d'une posture «normative » à une posture compréhensive et plurilingue, à co-construire des pistes d'enseignement explicite de la variation.

Nous pensons avec d'autres, que de «nouvelles méthodologies d'observation et d'analyse davantage collectives sont rendues nécessaires dans un contexte scolaire complexe, tant au regard des questions vives émergentes en didactique des langues, que des points aveugles dans

\footnotetext{
35 « Le processus inférentiel se présente ainsi comme un ensemble d'opérations logiques réalisées par les locuteurs et son aboutissement constitue une base pour l'interprétation de l'énoncé » (Chernyshova et Traverso, 2017 : 68)
} 
les praxéologies à l'œuvre » (Macaire, 2020). La recherche collaborative qui prend appui sur les discours et représentations des élèves et des enseignants via des outils réflexifs, des outils pédagogiques co-construits et une culture de l'observation partagée induit un changement de rôles, d'observés à observateurs, et permet d'envisager enfin les répertoires pluriels des élèves comme ressources pédagogiques, voire comme « savoir agir » et « savoir penser ».

\section{Références bibliographiques}

BAUTIER Élisabeth, 2004, «Les différentes dimensions de la maitrise de la langue et de leur évaluation », http://langage.ac-creteil.fr/IMG/pdf/dimensions_maitrise_langue_ evaluation_bautier.pdf

BAUTIER Élisabeth et GOIGOUX Roland, 2004, « Difficultés d'apprentissages, processus de secondarisation et pratiques enseignantes: une hypothèse relationnelle » Revue Française de Pédagogie, ${ }^{\circ} 148$, p. 89-100.

BARNĖCHE Sophie, 2005, «Vie urbaine et transmission des langues à Nouméa », Glottopol, $\mathrm{n}^{\circ} 5$, p. 67-91.

BECKERS Jacqueline et LEROY Charlène, 2011, «Quand un enseignant débutant se regarde enseigner... Description d'un dispositif mis en place dans le cadre du cours de didactique spéciale en psychologie et sciences de l'éducation », Puzzle. Centre Interfacultaire de Formation des enseignants, 29, p. 29-37.

BLANCHET Philippe, 2016, "Aux origines de la dynamique glottopolitique en faveur du provençal : un précurseur de l'approche polynomique ?» Marielle Rispail et Leila Messaoudi (dir) Des langues minoritaires en contexte plurilingue francophone, mélanges en hommage à Ahmed Boukous, Cahier de linguistique, 2016-42/1, p. 91-101.

BOUSQUET Vahimiti, 2018, Sur le chemin de l'école : à la croisée des parcours linguistiques et culturels des enseignants et des élèves, Portfolio DU Fles-Interculturalités, Université de la Nouvelle-Calédonie.

BUCHETON Dominique, 2009, L'agir enseignant: des gestes professionnels ajustés, Toulouse, Octarès Éditions.

CALVET Louis-Jean, 1998, Une ou des normes ? l'insécurité linguistique et normes endogènes en Afrique francophone, Paris, Didier Érudition, Collection « Langues et développement », p. 917.

CANDELIER Michel, 2003, «Evlang, l'éveil aux langues, une proposition originale pour la gestion du plurilinguisme en milieu scolaire », Contribution au Rapport mondial de l'UNESCO, construire des Sociétés du savoir, http://pluriliangues.univ-lemans.fr/

CHERNYSHOVA Elisaveta et TRAVERSO Véronique 2017, «Inférences et processus d'intercompréhension dans les interactions quotidiennes: quelques questions méthodologiques ", $\quad$ Cahiers de praxématique, $\mathrm{n}^{\circ} 68$, http://journals.openedition.org/praxematique/4536

CLERC-CONAN Stéphanie, RICHERME-MANCHET Claude, 2016, Didactique du français : pour une approche contextualisée et explicite de la langue à l'école, Eme Editions, Collection Proximités.

COLOMBEL Claire, 2012, Langues kanak, français, langues d'enseignement et de culture en Nouvelle- Calédonie: quelle glottopolitique pour quelle contextualisation sociodidactique?, Thèse de doctorat, Université d'Aix-Marseille.

COLOMBEL Claire, 2014, "Quel espace didactique possible pour les langues kanak?», Derivry-Plard M., Alao G. Yun-Roger S. et Suzuki E, La didactique plurilingue et pluriculturelle à l'épreuve du terrain éducatif, contraintes, résistance, tension, coll. PLID, Éditions des archives contemporaines : Paris, p. 127-136. 
COLOMBEL-TEUIRA Claire et FILLOL Véronique avec la collaboration de BOUSQUET Vahimiti, 2020, «Observer et objectiver le 'déjà-là' des élèves : premiers pas vers la bienvaillance linguistique? » Dinvaut, A. et Biichlë, L. (Dirs.), Mieux vivre en langues : la bientraitance linguistique, Editions 1'Harmattan, p. 237-253.

COLOMBEL-TEUIRA Claire et FILLOL Véronique, 2020, «Expériences de recherchescollaboratives au service de l'éducation en situation postcoloniale : enjeux, scientificité et légitimité ", Recherches en didactique des langues et des cultures, $\mathrm{n}^{\circ} 17-2$, consulté le 29 avril 2020, http://journals.openedition.org/rdlc/7619

COLOMBEL-TEUIRA Claire, 2019, « Je lis, j'écris le monde » : Développer des compétences littéraciques des élèves avec les langues océaniennes, Communication dans le cadre du COOL 11, 8 octobre 2019, Université de la Nouvelle-Calédonie.

COLOMBEL-TEUIRA Claire, FILLOL Véronique, GENEIX-RABAULT Stéphanie, VANDEPUTTE-TAVO Leslie, 2016, Les langues dans la ville : pratiques plurilingues et artistiques à Nouméa, Rapport de recherche, Nouméa, UNC-DGLFLF.

Commission du Grand débat sur l'avenir de l'école (2011) : Quelle école pour mon pays ? 60 recommandations pour la réussite de nos enfants.

CORTIER Claude et DI MEGLIO Alain, 2018, «Alterner les langues, translangager dans l'enseignement bilingue. Une question sociodidactique à partir du corse langue polynomique ", dans Erfurt J., Anna Weirich A., Carporal-Erbersold E., Educations plurilingues et pratiques langagières. Hommage à Christine Hélot, Peter Lang, p. 269290.

CORTIER Claude et DI MEGLIO Alain, 2007, « Pédagogie interactive, gestion plurilingue et rapport à la norme dans les écoles bilingues français-corse », Auzaneau, M. La mise en ceuvre des langues dans l'interaction », L'Harmattan, Parcours discursif, p. 198-216.

CUMMINS Jim, 1983, "Conceptual and Linguistic Foundations of Language Assessment », Stanley S. Seider (ed.) Issues of Language Assessment, Vol.II: Language Assessement and Curriculum Plannong, Illinois State Board of Education, Springfiels, p. 7-16.

FILLOL Véronique, 2020, «Quand les autobiographies langagières permettent l'analyse et le dépassement de l'insécurité linguistique », dans Valentin Feussi et Joanna Lorilleux (dirs.), (In)sécurité linguistique en francophonies. Perspectives in(ter)disciplinaires, Paris, L'Harmattan, Espaces Discursifs, p. 141-153.

FILLOL Véronique, 2016a, « Le français « langue partagée » en Nouvelle-Calédonie : Quelle place pour la diversité linguistique à l'école? Quelle didactique du français contextualisée ?», Bertucci M.-M., Les français régionaux, éditions Peter Lang, Collection « Langue, plurilinguisme, changement social », p. 187-200.

FILLOL Véronique, 2016b, «Les biographies langagières comme outil de lecture de la situation postcoloniale et comme outil d'empowerment dans une démarche sociodidactique », Contextes et Didactique, n8, p. 9-22.

FILLOL Véronique, 2009, «Des langues, du rapport au langage et aux apprentissages pour réussir à l'école ", Vernaudon J. et Fillol V., Vers une école plurilingue dans les collectivités françaises d'Océanie et de Guyane, Paris, L'Harmattan, p. 137-154.

FILLOL Véronique et COLOMBEL Claire, 2014, «Quelles politiques linguistiques éducatives pour la Nouvelle-Calédonie? Quels dispositifs d'enseignement des langues? Réflexions sociodidactiques », dans I. Nocus, J. Vernaudon et M. Paia (eds), Apprendre plusieurs langues, plusieurs langues pour apprendre. L'école plurilingue en Outre-mer, Presses Universitaires de Rennes, Collection «Des sociétés », p. 199-221.

FILLOL Véronique, GENEIX-RABAULT Stéphanie et RAZAFIMANDIMBIMANANA Elatiana, 2018, " Approches inclusives des pluralités et formation à/par la réflexivité : une expérience en contexte multilingue », Les journées de la recherche en éducation, Tahiti, Université de la Polynésie française, 3-4 mai. 
FILLOL Véronique et VERNAUDON Jacques, 2004, «Les langues Kanak et le français : langues d'enseignement et de culture en Nouvelle-Calédonie. D'un compromis à un bilinguisme équilibré ?», ELA, n 133 , p. 57-67.

MACAIRE Dominique, 2020, "La « recherche-formation », une contribution aux approches collaboratives en formation initiale d'enseignants de langues ", Recherches en didactique des langues et des cultures, $\mathrm{n}^{\circ} 17-2$, consulté le 29 avril 2020, http://journals.openedition.org/rdlc/7697.

MARCELLESI Jean-Baptiste, 1983, «La définition des langues en domaine roman: les enseignements à tirer de la situation corse ", dans Actes du congrès des romanistes d'Aix-en-Provence vol. $\mathrm{n}^{\mathrm{0}}$ 5, Sociolinguistique des langues romanes.

MOREL Françoise, BUCHETON Dominique, CARAYON Brigitte, FAUCANIÉ Hélène et LAUX Sandrine, 2015, "Décrire les gestes professionnels pour comprendre des pratiques efficientes », Le français aujourd'hui, $\mathrm{n}^{\circ} 188$, p. 65-77.

MOREL-LAB, Anne, 2014, Les passeurs de langues, acteurs de l'interculturel en milieu professionnel plurilingue. Le cas des projets miniers en Nouvelle-Calédonie, Thèse en Sciences du langage, Université de la Nouvelle-Calédonie.

MOSELEY Christopher, 2010, Atlas des langues en danger dans le monde, 3 ème édition. Paris, Editions UNESCO. Version en ligne : http://www.unesco.org/culture/en/endangeredlanguages/atlas

OLIVESI Stéphane, 2012, « Observer l'autoscopie », Communication (en ligne, non paginé), Vol. 30/2, https://journals.openedition.org/communication/3501

RAZAFI Elatiana et FAVARD Nicolas, 2018, " Les élèves aux besoins éducatifs particuliers se mettent en représentation: 'on parle plusieurs langues pour progresser' ». Dans Enseignement et formation du/en français en contexte plurilingue, Hanoï, Édition de 1'Université nationale du Vietnam à Hanoï, p. 311-335.

ROBERT Stéphane, 2003, "Introduction : de la grammaticalisation à la transcatégorialité », dans S. Robert (éd.), Afrique et Langage $\mathrm{n}^{\circ} 5$ : Perspectives synchroniques sur la grammaticalisation. Polysémie, transcatégorialité et échelles syntaxiques, Louvain/Paris : Peeters, 9-18.

SORBA Nicolas, 2016, Évolution du concept de langue polynomique au sein de la société corse, Thèse de doctorat en cultures et langues régionales, Université de Corse.

VERNAUDON Jacques, 2020, «Les langues polynésiennes et kanak, des «langues de France » en contexte de décolonisation », Glottopol, $\mathrm{n}^{\circ} 34$, juillet 2020 : http://glottopol.univ-rouen.fr/numero_34.html\#res_vernaudon.

VERNAUDON Jacques, 2009, «Observation réfléchie et comparée des langues océaniennes er de la langue française ", dans Vernaudon, J. et Fillol, V. Vers une école plurilingue dans les collectivités françaises d'Océanie et de Guyane, Paris, L'Harmattan, p. 191206.

\section{Convention de transcription}

\section{Tours de paroles}

[ interruption et chevauchement, le crochet apparait sur chacune des deux lignes.

] Fin du chevauchement si un des interlocuteurs ne s'est pas interrompu

$=\quad$ Enchainement immédiat entre deux tours

\section{Silences et pauses}

(.) Pause (dans le tour d'un locuteur) inférieure à 1 seconde 
(2') Pause chronométrée supérieure à 1 seconde

(silence) Pause entre les prises de parole de deux ou plusieurs interlocuteurs, chronométrée ou non

\section{Rythmes}

$\begin{array}{ll}6 & \text { Chute d'un son } \\ : & \text { Allongement d'un son } \\ :: . & \text { Allongement important d'un son } \\ - & \text { Mot brutalement interrompu } \\ - & \text { Liaison non faite }\end{array}$

Voix et intonations

/ Intonation légèrement montante

$\uparrow \quad$ Intonation fortement montante

1 Intonation légèrement descendante

$\downarrow \quad$ Intonation fortement descendante

(FORT) ...+ Caractéristiques vocales, le signe + indique la fin

NON Les mots en majuscules indiquent l'insistance et l'emphase

\section{Actions et gestes}

(se tourne) Les actions et gestes sont notés entre parenthèses

\section{Unités non lexicales}

$\begin{array}{ll}\text { (ASP) } & \text { Aspiration } \\ \text { (SP) } & \text { Soupir } \\ \text { (rire) } & \text { Note un rire }\end{array}$

Indications « méta »

[...] Coupure due au transcripteur

XXX inaudible 


\section{GLOTTOPOL}

Revue de sociolinguistique en ligne

Comité de rédaction : Michaël Abecassis (University of Oxford), Salih Akin (Université de Rouen Normandie), Sophie Babault (Université de Lille), Aude Bretegnier (Université du Mans), Claude Caitucoli, Véronique Castellotti (Université de Tours), Régine DelamotteLegrand (Université de Rouen Normandie), Alexandre Duchêne (Université de Fribourg), Valentin Feussi (Université d'Angers), Robert Fournier (Carleton University, Ottawa), Stéphanie Galligani (Université Grenoble Alpes), Médéric Gasquet-Cyrus (Université AixMarseille), Emmanuelle Huver (Université de Tours), Normand Labrie (Université de Toronto), Foued Laroussi (Université de Rouen Normandie), Benoit Leblanc (Université du Québec à Trois-Rivières), Mylène Lebon-Eyquem (Université de la Réunion), Fabienne Leconte (Université de Rouen Normandie), Gudrun Ledegen (Université de Rennes), Danièle Moore (Simon Fraser University, Vancouver), Clara Mortamet (Université Jean Monnet, Saint Etienne), Alioune Ndao (Université Cheik Anta Diop, Dakar), Isabelle Pierozak (Université de Tours), Cécile Van den Avenne (Université Sorbonne Nouvelle, Paris 3).

Rédactrice en chef : Clara Mortamet.

Directrice de publication : Fabienne Leconte.

Comité scientifique: Michelle Auzanneau (Université de Paris), Margaret Bento (Université de Paris), Jacqueline Billiez (Université de Grenobles Alpes), Philippe Blanchet (Université de Rennes), Jean-Michel Eloy (Université d'Amiens), Françoise Gadet (Université Paris Nanterre), Monica Heller (Université de Moncton), Caroline Juillard (Université de Paris), Jean-Marie Klinkenberg (Université de Liège), Marinette Matthey (Université Grenoble Alpes), Marie-Louise Moreau (Université de Mons-Hainault), Robert Nicolaï (Université Côte d'Azur), Didier de Robillard (Université de Tours), Valérie Spaëth (Université Sorbonne nouvelle), Claude Truchot (Université de Strasbourg), Daniel Véronique (Université AixMarseille).

\section{Comité de lecture pour ce numéro :}

Nathalie Auger, Michèle Auzanneau, Margaret Bento, Stéphane Bonnery, Josiane Boutet, Lucile Cadet, Danièle Cogis, Claudine Garcia-Debanc, Marc Debono Régine Delamotte, JeanFrançois De Pietro, Marie-Laure Elalouf, Valentin Feussi, Laurent Gajo, Emmanuelle Huver, Christian Lagarde, Mylène Lebon-Eyquem, Fabienne Leconte, Gudrun Ledegen, Nadia Maillard, Maira Mamede, Bruno Maurer, Clara Mortamet, Fanny Rink, Valerie Spaëth, Marielle Rispail, Françoise Ropé, Eguzki Urteaga, Cécile Van den Avenne, Daniel Véronique 